Check for updates

Cite this: RSC Adv., 2018, 8, 33025

Received 1st June 2018

Accepted 4th September 2018

DOI: $10.1039 / c 8 r a 04695 f$

rsc.li/rsc-advances

\section{Effect of phosphorus content on mechanical properties of polymeric nickel composite materials with a diamond-structure microlattice}

\author{
Yungang Zhao, ${ }^{\text {ab }}$ Fang Wei, (D) ${ }^{a}$ Xinqing Zhao, ${ }^{* b}$ Qinglin Yang, (DD *a Lin Guo (D) a \\ and Lei Jiang ${ }^{a}$
}

Periodical and ordered polymer-nickel-coated composite materials with a diamond-structure microlattice and various contents of phosphorus (4.10 wt\%, $8.01 \mathrm{wt} \%, 12.25 \mathrm{wt} \%, 16.08 \mathrm{wt} \%, 20.21 \mathrm{wt} \%$ ) were fabricated via electroless nickel-phosphorus ( $\mathrm{Ni}-\mathrm{P})$ coating onto diamond-structured polymeric templates using a 3D printing stereo lithography apparatus. With the increase in $\mathrm{P}$ content, the crystal morphology transfers from crystal to non-crystal. By controlling identical $1.0 \mu \mathrm{m}$-thickness of 5 different content coatings onto templates, the properties of 5 different microlattice composites were tested by uniaxial compression. To confirm the thickness and $\mathrm{P}$ content, several mathematical models were developed to direct the subsequent experiments and all theoretical predictions are in agreement with factual characterization. The composite with $8.01 \mathrm{wt} \%$ phosphorus content and density of $240.4 \mathrm{~kg} \mathrm{~m}^{-3}$ performs best, with the maximum compressive strength reaches $1.08 \mathrm{MPa}$, which is 2.1 times higher than that of polymer templates.

\section{Introduction}

Cellular materials are noted for their low densities as well as unique mechanical, electrical, thermal, acoustic, and ultrasonic properties. However, most cellular materials, such as foams and aerogels, will present imperfections due to their stochastic spatial configuration; hence, lattice materials can be considered as promising alternatives to metallic foams in lightweight structures. ${ }^{1}$ For example, the ordered and periodical lightweight cellular materials, namely, microlattices, can be considered as perfect alternatives since they possess specific properties such as excellent noise reduction, ${ }^{5}$ good energy storage, ${ }^{6}$ effective thermal conductivity, ${ }^{7}$ and strong energy absorption, ${ }^{8,9}$ thus presenting potential applications in aviation, aerospace and architecture. $^{2-4}$

The properties of ordered microlattice materials depend not only on the architecture but also on the solid constituents. ${ }^{10}$ Ordered lattice-type architectures including tetrahedral lattice, octahedral lattice, vertebral lattice, and diamond lattice can exhibit the merits of stretching-dominated properties. ${ }^{11}$ Moreover, hollow-tube microlattices with octahedral structure, which are fabricated by self-propagating photopolymer waveguide prototyping, present high structural stability and recovery. ${ }^{3}$ Compared with octahedral lattices, the diamond lattice as the microlattice cell exhibits low density and high capacity to withstand compression, and it should perform much preferably

${ }^{a}$ School of Chemistry, Beihang University, China. E-mail: yangql@buaa.edu.cn

${ }^{b}$ School of Material Science and Engineering, Beihang University, China. E-mail: xinqing@buaa.edu.cn and effectively for applications in uniaxial compressions. ${ }^{12}$ Alternatively, we employed 3D printing technique, which highlights the significance of the processing in high controllability and designability of the overall structure. With regard to the solid constituents, electroless composite plating is a wellestablished strategy for template coating, which is more efficient in improving the mechanical properties. The amount of phosphorus added can be high, medium, and low. The change in phosphorus content would certainly influence crystal morphology, thus inducing variations in properties, ${ }^{13}$ particularly mechanical properties. The crystal morphology of Ni-P coatings changes gradually from crystal to non-crystal type with the increase in phosphorus content. ${ }^{14,15}$ In other words, crystal transformation will definitely induce changes in the mechanical properties. ${ }^{16}$ The attempt to systematically investigate the Ni-P coatings with low, medium, and high phosphorus content on three-dimensional microlattice will promisingly accelerate the investigation and popularization of microlattices.

Herein, polymer-Ni-P microlattice composite materials with different phosphorus contents were fabricated and the corresponding mathematical models were induced from experimental data for direction of the subsequent experiments to control the thickness and phosphorus contents of Ni-P coatings. Inspired by previous related results, ${ }^{17-20}$ lightweight composites of polymer-Ni-P microlattice with five different contents of phosphorus $(4.10 \mathrm{wt} \%, 8.01 \mathrm{wt} \%, 12.25 \mathrm{wt} \%$, $16.08 \mathrm{wt} \%, 20.21 \mathrm{wt} \%)$ were fabricated. The diamond samples were obtained by first using a polymeric template fabricated by 3D printing and then coating the template with Ni-P by electroless plating. The surface characterization, crystal 
morphology and mechanical properties of the diamondstructure polymer-Ni-P microlattice with different phosphorous content were investigated.

\section{Experimental}

All reagents were of analytical grade and employed without further purification. Sodium hydroxide $(\mathrm{NaOH}, \mathrm{AR}, 99 \%)$, potassium permanganate $\left(\mathrm{KMnO}_{4}, \mathrm{AR}, 99 \%\right)$, acetone $\left(\mathrm{CH}_{3}{ }^{-}\right.$ $\left.\mathrm{COCH}_{3}, \mathrm{AR}, 99 \%\right)$, stannous chloride $\left(\mathrm{SnCl}_{2} \cdot 2 \mathrm{H}_{2} \mathrm{O}, \mathrm{AR}, 99 \%\right)$, and palladium chloride $\left(\mathrm{PdCl}_{2}, \mathrm{AR}, 99 \%\right)$ were purchased from Alfa Aesar. The plating solution was a commercial solution purchased from DSE Technology Co., Ltd. Templates were manufactured in Yiwu Realmaker Electronic Technology Co. Ltd. Deionized water used in experiments was purified using a laboratory water purification system $(18.25 \mathrm{M} \Omega \mathrm{cm})$.

Microlattices with diamond structure were designed using the computer-aided design (CAD) software (Solid Works) and manufactured by 3D printing. A 3D printer (MiiCraft, Young Optics Inc) based on stereolithography was used in this process. The stereolithography resin used in this study was an acrylatebased EX-200 resin (Yiwu Realmaker Electronic Technology Co. Ltd). The size of the resulting 3D microlattice was approximately $3.0 \times 2.0 \times 1.0 \mathrm{~cm}^{3}$.

The microlattice templates were cleaned successively with acetone and ethanol for $15 \mathrm{~min}$. Then, the templates were immersed into a solution of $\mathrm{KMnO}_{4}\left(0.40 \mathrm{~mol} \mathrm{~L}^{-1}\right)$ and $\mathrm{NaOH}$ $\left(0.75 \mathrm{~mol} \mathrm{~L}^{-1}\right)$ at $30{ }^{\circ} \mathrm{C}$ for $20 \mathrm{~min}$ to roughen the surface microscopically, following which the templates were immersed in a neutralizing solution $\left(\mathrm{H}_{2} \mathrm{C}_{2} \mathrm{O}_{4} \cdot \mathrm{H}_{2} \mathrm{O}, 0.22 \mathrm{~mol} \mathrm{~L}^{-1}\right)$ at $30^{\circ} \mathrm{C}$ for $10 \mathrm{~min}$ to remove residual $\mathrm{MnO}_{2}$. The surface activation process of the templates was performed via dipping them into $10 \%$ hydrochloric acid $(\mathrm{HCl})$ at $30^{\circ} \mathrm{C}$ for $5 \mathrm{~min}$ for acidifying the surface and then into $0.16 \mathrm{~mol} \mathrm{~L}^{-1}$ stannous chloride solution $\left(\mathrm{SnCl}_{2}\right)$. After hydrolysis in deionized water, the surface sensitization process of the templates was conducted by immersion of the samples into $0.23 \mathrm{mmol} \mathrm{L}{ }^{-1}$ palladium chloride $\left(\mathrm{PdCl}_{2}\right)$ solution at $30{ }^{\circ} \mathrm{C}$ for $5 \mathrm{~min}$. Deionized water was used to wash the templates after every step. Finally, the templates were dried in a drying oven at $80^{\circ} \mathrm{C}$ for $6 \mathrm{~h}$.

The deposition of Ni-P coatings was performed at $70^{\circ} \mathrm{C}$ with $\mathrm{pH} 8.5$, and the phosphorus content was controlled by adjusting the ratio of $\mathrm{NaH}_{2} \mathrm{PO}_{2}$ to $\mathrm{Ni}_{2} \mathrm{SO}_{4}$. Mathematical formula was developed by initially implement of phosphorus content to several ratios to predict all trends of phosphorus content to ratio and every ratio would repeat 3 times to induce a simple regularity respectively. Finally, the confirmed phosphorus contents deduced by the model would be calculated, and the thickness of coatings was determined and similarly controlled by applying the plating time suggested by another five parabolic mathematical models. Composites of the confirmed phosphorus content and thickness with diamond structure were applied for mechanical test.

The morphology and the thickness of $\mathrm{Ni}-\mathrm{P}$ films were observed using an environmental scanning electron microscope (ESEM, FEI Quanta-250 FEG). The crystal structure of the resulting Ni-P coatings was characterized using a X-ray diffractometer (XRD-1200, Rigaku Denki Co. Ltd., Japan) under the following conditions: $\mathrm{Cu}$ Ka ray $(\lambda=1.5406 \AA)$ for the radiation source, voltage of $40 \mathrm{kV}$, current of $30 \mathrm{~mA}$, scanning angle range $(2 \theta)$ of $10-80^{\circ}$, and scanning step of $0.02^{\circ}$.

The surface morphology of the microlattice materials was analyzed using a X-ray energy-dispersive spectrometer (EDS). The mass of the samples was measured using an electronic balance (Sartorius BS 110S, Sartorius BS). The speed of the compressive test was controlled at $0.5 \mathrm{~mm} \mathrm{~min}^{-1}$ for all microlattice samples using INSTRON-5565.

\section{Results and discussion}

Fig. 1 shows the fabrication of the composite microlattice. Fig. 1A and B clearly exhibit the diamond-structure microlattice macrostructure of the 3D-printed polymer template and the resulting materials, respectively. The average diameter of the cross-section strut is about $285.7 \mu \mathrm{m}$ (Fig. 1D) and the thickness of the thin film is about $2 \mu \mathrm{m}$ (Fig. 1C). The effects of 14 different ratios of solution A to B, with 3 repetitions of the same ratio (marked I, II, III for accuracy), on phosphorus content in $\mathrm{Ni}-\mathrm{P}$ coatings were involved in developing the key mathematical model. The data mentioned above are presented by fitting cure and average content in Fig. 2, and the corresponding mathematical model of the fitting curve was deduced by the average phosphorus content.

The equation of the deduced curve is presented for further investigation of relation between solution ratio and phosphorus content. $C$ scales nonlinearly with $r$, and exhibits a scale of

$$
C=22.21-24.43 \mathrm{e}^{-0.106 r}
$$

where $C$ is the phosphorus content and the factor $r$ represents the ratio of solution A to B. When $C$ is equal to 4.0, 8.0, 12.0, 16.0 , and 20.0, the corresponding $r$ is $2.8,5.1,8.2,12.9$, and 22.3. On rounding off for convenience of subsequent experiments, $C$ is equal to $3.0,5.0,9.0,13.0$, and 22.0. Then, by

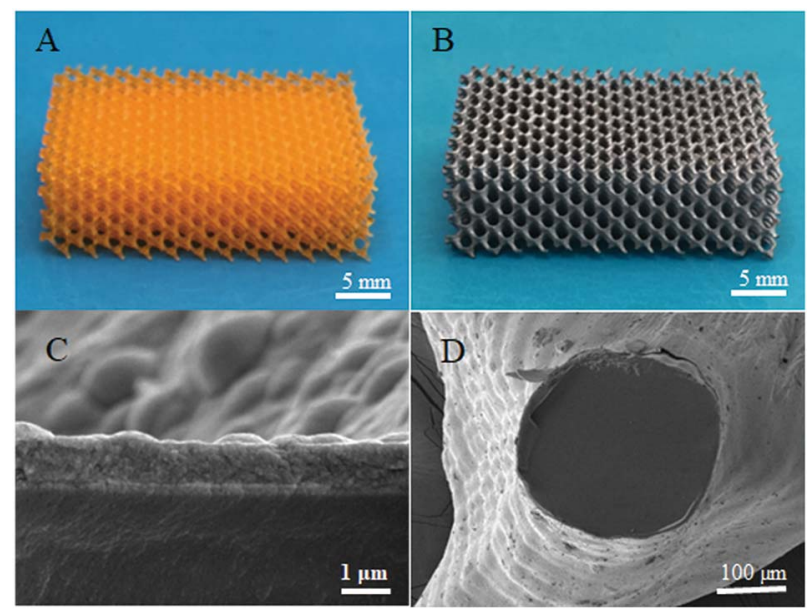

Fig. 1 Fabrication and characterization of microlattice composites with diamond structure. (A) Polymeric template of diamond structure; (B) polymer-Ni-P microlattice with 8.01 wt\% P content; (C) SEM image of cross-section; (D) SEM image of the unit cell. 


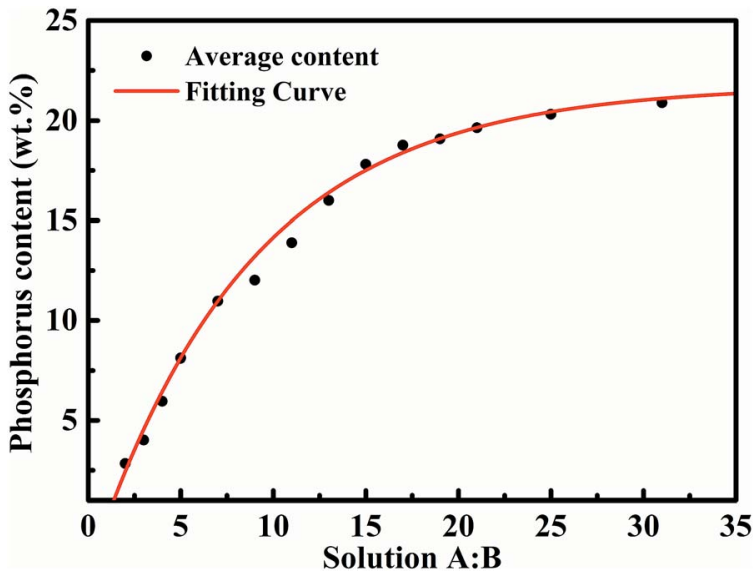

Fig. 2 Fitting curve of phosphorus content varying with the ratio of solution $A$ to $B$.

substituting the corresponding $C$ value into the above equation, the corresponding $r$ value can be calculated. The corresponding targeted phosphorus contents will be acquired according to the corresponding $r$ values in the subsequent experiments. By the direction of corresponding $r$ values, five actual phosphorus contents obtained from the five different ratios, 0.0, 5.0, 9.0, 13.0, and 22.0, and the actual results correspond well, as listed in Table 1. III was elected for most approximating the average phosphorus contents and characterized in Fig. 1. When the settled phosphorus content was determined, thickness of every phosphorus content was still uncertainty.

Herein, for comparison of mechanical properties with different phosphorus contents, the thickness of the coating was further investigated to study the systematic mechanical properties. Then, the process of electroless plating was performed in different time periods ( $5 \mathrm{~min}, 10 \mathrm{~min}, 15 \mathrm{~min}$ ), and the corresponding thicknesses of the coatings were measured by SEM, as presented in Fig. 3. Then, parabolic mathematical formulas of five different phosphorus contents are induced for theoretical time (when the film thickness is $1.0 \mu \mathrm{m}$ ) are calculated by parabolic mathematical models presented in Table 2. Fig. 3 briefly shows the change in trend of thickness for all phosphorus contents, and the actual content corresponds well with expected values. Combining with the equation mentioned above and models in Table 2, different but definite phosphorus contents and identical thickness $(1.0 \mu \mathrm{m})$ can be complemented simultaneously by the equation mentioned above and models in Table 2.

Table 1 Actual phosphorous contents obtained from five different solution ratios of $\mathrm{NaH}_{2} \mathrm{PO}_{2}$ to $\mathrm{Ni}_{2} \mathrm{SO}_{4}$ by the mathematical model

\begin{tabular}{lcccc}
\hline \multicolumn{5}{c}{ Phosphorous content } \\
\cline { 2 - 5 } $\mathrm{NaH}_{2} \mathrm{PO}_{2} / \mathrm{Ni}_{2} \mathrm{SO}_{4}$ & $\mathrm{I}(\mathrm{wt} \%)$ & $\mathrm{II}(\mathrm{wt} \%)$ & $\mathrm{III}(\mathrm{wt} \%)$ & Average (wt\%) \\
\hline $3: 1$ & 3.89 & 4.17 & 4.10 & 4.05 \\
$5: 1$ & 8.30 & 7.94 & 8.01 & 8.08 \\
$9: 1$ & 12.16 & 11.99 & 12.25 & 12.13 \\
$13: 1$ & 15.97 & 16.13 & 16.08 & 16.06 \\
$22: 1$ & 20.01 & 19.88 & 20.21 & 20.03
\end{tabular}

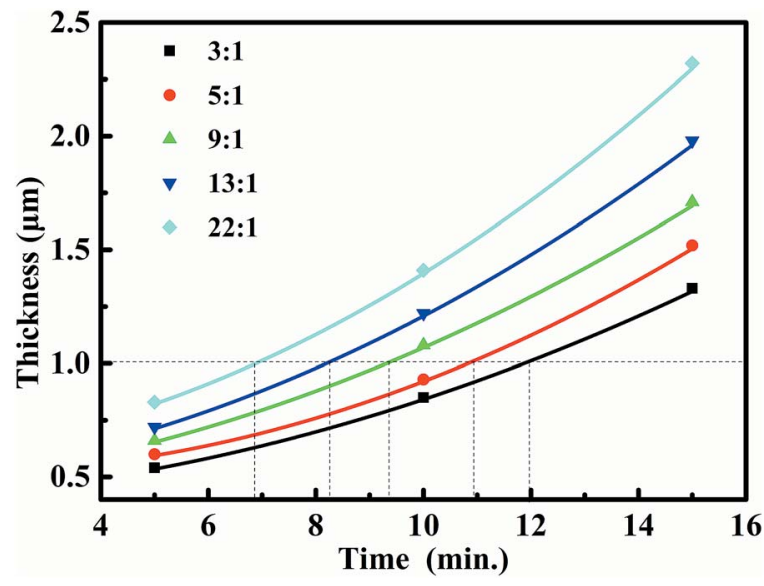

Fig. 3 Trend of electroless plating thickness to time corresponding to five different phosphorus contents.

Fig. 4 shows that the characteristic peaks of Ni-P coatings gradually transit from spikes to a broad diffraction peak, that is, the crystal morphology transforms from crystal to non-crystal. When the phosphorous content is below $12.25 \mathrm{wt} \%$, diffraction peaks at around $2 \theta=44.8^{\circ}$ reveal the crystal of $\mathrm{Ni}$ and the peaks broaden with the increase in phosphorous content. Films with phosphorous content of 16.08 and $20.21 \mathrm{wt} \%$ present similar broad peaks, indicating amorphous phase. This occurs because the increase in phosphorus segregation on nickel prevents the nucleation of the face centered cubic (FCC) Ni phase in $<111>$ plane, which eventually results in an amorphous structure.

Then, characterization and comparison of microlattice composites in five confirmed phosphorus contents are presented in Fig. 5 and 6. Fig. 5 shows the surface morphology and film thickness of the microlattice composite materials with different phosphorus contents $(4.10 \mathrm{wt} \%, 8.01 \mathrm{wt} \%, 12.25 \mathrm{wt} \%$, $16.08 \mathrm{wt} \%$, and $20.21 \mathrm{wt} \%$ ) and Fig. 6 exhibits the mechanical trends of microlattice composites in different phosphorus contents. The surface morphology of Ni-P coatings exhibited a nodular feature with a typical electroless structure, and top right insets indicate the approach of wall thickness to $1.0 \mu \mathrm{m}$. The Ni-P coatings have clearly presented a trend of change in surface morphology and their nodule sizes decreased clearly with the increase in phosphorus content, which correspond well with the already presented studies. ${ }^{21}$ This could be attributed to the crystalline nature of films. It has been demonstrated that lattice materials become increasingly strained and grain sizes decrease with the increase in phosphorus concentration. ${ }^{22}$ Films with high phosphorous content presenting smooth and uniform morphology indicated the amorphous nature as also revealed during XRD analysis (Fig. 4). The existing grains of films in low phosphorous content may induce bigger nodule sizes than those of high phosphorous content, which was also confirmed by a similar investigation. ${ }^{13}$

It can be observed from Fig. 6 that when the surface of the polymer was deposited with $\mathrm{Ni}-\mathrm{P}$ coatings, the mechanical strength will certainly increase. The first point in Fig. 6 represents the maximum stress of polymer lattice template. With the 
Table 2 Mathematical model of thickness in different ratio

\begin{tabular}{llrl}
\hline $\mathrm{NaH}_{2} \mathrm{PO}_{2} / \mathrm{Ni}_{2} \mathrm{SO}_{4}$ & Mathematical model $(T$ for thickness, $t$ for time $)$ & Theoretical time & Actual thickness \\
\hline $3: 1$ & $T=0.0036 t^{2}+0.0082 t+0.409$ & 11.78 & 1.08 \\
$5: 1$ & $T=0.0052 t^{2}-0.0121 t+0.527$ & 10.76 & 0.99 \\
$9: 1$ & $T=0.0041 t^{2}+0.0237 t+0.319$ & 10.35 & 1.06 \\
$13: 1$ & $T=0.0051 t^{2}+0.0253 t+0.465$ & 8.08 & 1.04 \\
$22: 1$ & $T=0.0064 t^{2}+0.0211 t+0.565$ & 6.77 & 0.97
\end{tabular}

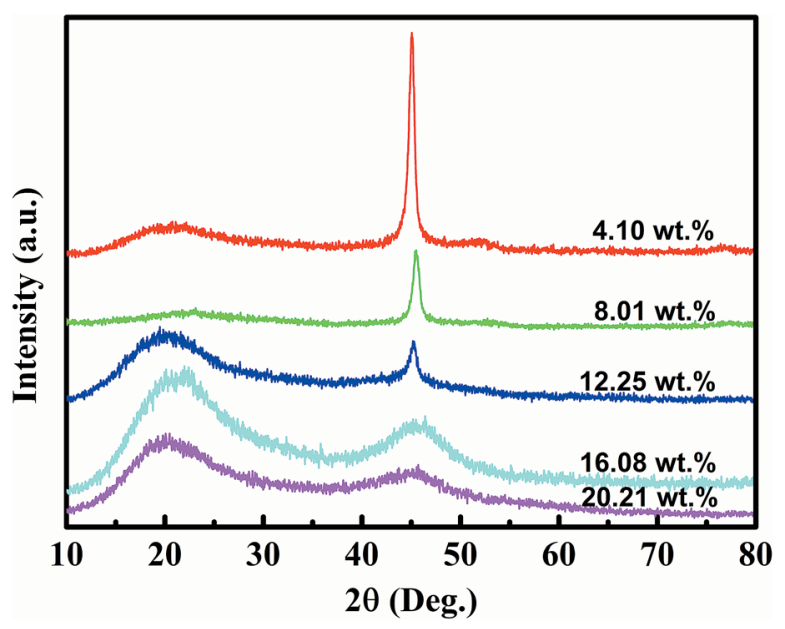

Fig. 4 The XRD patterns of $\mathrm{Ni}-\mathrm{P}$ coatings with different phosphorus contents $(4.10,8.01,12.25,16.08$, and 20.21 wt $\%)$.

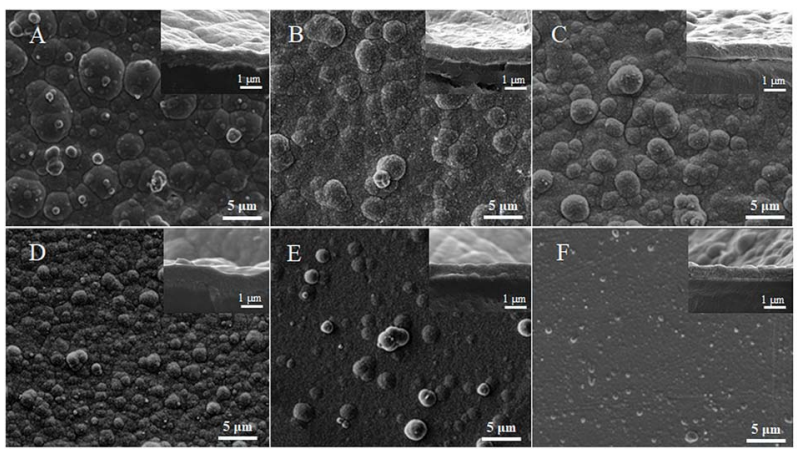

Fig. 5 The surface morphology of $\mathrm{Ni}-\mathrm{P}$ coatings with different phosphorus contents. (A-F) represent phosphorus contents of 4.10 , $8.01,12.25,16.08,20.21$ and $20.90 \mathrm{wt} \%$, respectively.

increase in phosphorus content until $8.01 \mathrm{wt} \%$, the maximum stress increased to $1.08 \mathrm{MPa}$; then an opposite trend was observed because of the transition to the amorphous phase. According to XRD analysis, when the phosphorus content increased up to $8.01 \mathrm{wt} \%$, the crystalline structure still existed, but in supersaturation, which induced lattice distortion from dislocation of $\mathrm{Ni}$ and $\mathrm{P}$ atoms. The lattice distortion would bring about stresses among grain boundaries that increase the strength and then improve the property of compression with the increase in phosphorus content until $8.01 \mathrm{wt} \%$ up to $1.08 \mathrm{MPa}$. When the phosphorus content reached more than $8.01 \mathrm{wt} \%$, increasing amorphous phase existed. However, although the mechanical properties are supposed to increase

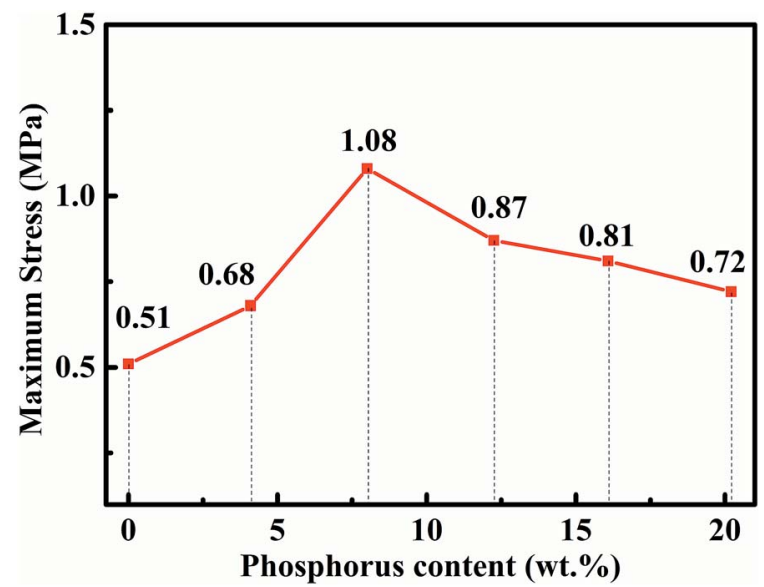

Fig. 6 Plot representing the trend of stress of microlattice composites to phosphorus contents.

for rigid amorphous phase, the diamond-structure microlattice when compressed would induce internal stretching stress, by which the amorphous phase would present brittleness. Therefore, if phosphorus content increased further, the property of compression would worsen than before, as presented in Fig. 6 . Fig. 6 also shows that when controlling the thickness to around $1 \mu \mathrm{m}$, the polymer-Ni-P microlattice composites with the phosphorus content of $8.01 \mathrm{wt} \%$ perform better than other microlattices, and the corresponding parameters are as follows: the thickness of films is $0.99 \mu \mathrm{m}$, the density is $240.4 \mathrm{~kg} \mathrm{~m}^{-3}$ (which belongs to lightweight strategy), and the maximum compressive strength is $1.08 \mathrm{MPa}$. Compared with the polymeric microlattice, the maximum mechanical strength of composites with diamond structure is improved by 2.1 times.

\section{Conclusions}

A feasible method for the fabrication of lightweight diamondstructured polymer-Ni-P microlattice composite materials with different phosphorus contents has been developed via combining SLA with electroless plating. The Ni-P coatings enhanced the mechanical properties of polymeric microlattices, and the composites perform best (by 2.1 times) with a phosphorus content of $8.01 \mathrm{wt} \%$. The corresponding parameters are as follows: the film thickness is $0.99 \mu \mathrm{m}$, the density is $240.4 \mathrm{~kg}$ $\mathrm{m}^{-3}$, and the maximum compressive strength is $1.08 \mathrm{MPa}$. More critically, the process of confirming the targeted thickness and phosphorus content by the mathematical models is an innovative approach for application in related fields. 


\section{Conflicts of interest}

There are no conflicts to declare.

\section{Acknowledgements}

The authors gratefully acknowledge the financial support provided by the National Natural Science Foundation of China (Project Number: 51372010).

\section{Notes and references}

1 V. S. Deshpande, N. A. Fleck and M. F. Ashby, J. Mech. Phys. Solids, 2001, 49, 1747-1769.

2 L. R. Meza, S. Das and J. R. Greer, Science, 2014, 345, 13221326.

3 T. A. Schaedler, A. J. Jacobsen, A. Torrents, A. E. Sorensen, J. Lian, J. R. Greer, L. Valdevit and W. B. Carter, Science, 2011, 334, 962-964.

4 N. T. Trifale, E. A. Nauman and K. Yazawa, ACS Appl. Mater. Interfaces, 2016, 8, 35534-35544.

5 Z. Xu, H. Sun and C. Gao, APL Mater., 2013, 1, 030901.

6 L. Salari-Sharif, T. A. Schaedler and L. Valdevit, J. Mater. Res., 2014, 29, 1755-1770.

7 L. Valdevit, A. Pantano, H. A. Stone and A. G. Evans, Int. J. Heat Mass Transfer, 2006, 49, 3819-3830.

8 Z. Liu, W. Chen, J. Carstensen, J. Ketkaew, R. M. O. Mota, J. K. Guest and J. Schroers, Acta Mater., 2016, 105, 35-43.
9 K. J. Maloney, C. S. Roper, A. J. Jacobsen, W. B. Carter, L. Valdevit and T. A. Schaedler, APL Mater., 2013, 1, 022106.

10 A. Torrents, T. A. Schaedler, A. J. Jacobsen, W. B. Carter and L. Valdevit, Acta Mater., 2012, 60, 3511-3523.

11 V. S. Deshpande, M. F. Ashby and N. A. Fleck, Acta Mater., 2001, 49, 1035-1040.

12 H. L. Fan, D. N. Fang and F. N. Jing, Mater. Des., 2008, 29, 2038-2042.

13 J. W. Yoon, J. H. Park, C. C. Shur and S. B. Jung, Microelectron. Eng., 2007, 84, 2552-2557.

14 K. G. Keong and S. Malinov, Acta Metall. Sin., 2001, 37, 419424.

15 W. Sha, X. Wu and K. G. Keong, Crystallisation of nickelphosphorus (Ni-P) deposits with high phosphorus content, 2011.

16 C. C. Hu and A. Bai, Mater. Chem. Phys., 2003, 77, 215-225.

17 J. Xu, Y. Gao, H. Huang, Q. Yang, L. Guo and L. Jiang, Sci. China: Chem., 2016, 59, 1632-1637.

18 Y. F. Zhou, C. Z. Yao, Q. L. Yang, L. Guo and L. Jiang, Adv. Eng. Mater., 2016, 18, 236-240.

19 F. Wei, Y. Zhou, Q. Yang, L. Guo and L. Jiang, RSC Adv., 2017, 7, 54668-54673.

20 Q. Fan, Y. Gao, Y. Zhao, Q. Yang, L. Guo and L. Jiang, Mater. Lett., 2018, 215, 242-245.

21 S. M. A. Shibli and V. S. Dilimon, Int. J. Hydrogen Energy, 2007, 32, 1694-1700.

22 K. H. Hur, J. H. Jeong and N. L. Dong, J. Mater. Sci., 1990, 25, 2573-2584. 